\title{
Polarization-sensitive interferometric synthetic aperture microscopy
}

\author{
Fredrick A. South, ${ }^{1,2}$ Yuan-Zhi Liu, ${ }^{1,2}$ Yang Xu, ${ }^{1,2}$ Nathan D. Shemonski, ${ }^{1,2}$ \\ P. Scott Carney, ${ }^{1,2}$ and Stephen A. Boppart ${ }^{1,2,3}$ \\ ${ }^{1}$ Beckman Institute for Advanced Science and Technology, University of Illinois at Urbana-Champaign, \\ Urbana, Illinois 61801, USA \\ ${ }^{2}$ Department of Electrical and Computer Engineering, University of Illinois at Urbana-Champaign, Urbana, \\ Illinois 61801, USA \\ ${ }^{3}$ Departments of Bioengineering and Internal Medicine, University of Illinois at Urbana-Champaign, Urbana, \\ Illinois 61801, USA
}

(Received 21 September 2015; accepted 10 November 2015; published online 23 November 2015)

\begin{abstract}
Three-dimensional optical microscopy suffers from the well-known compromise between transverse resolution and depth-of-field. This is true for both structural imaging methods and their functional extensions. Interferometric synthetic aperture microscopy (ISAM) is a solution to the 3D coherent microscopy inverse problem that provides depth-independent transverse resolution. We demonstrate the extension of ISAM to polarization sensitive imaging, termed polarization-sensitive interferometric synthetic aperture microscopy (PS-ISAM). This technique is the first functionalization of the ISAM method and provides improved depth-of-field for polarization-sensitive imaging. The basic assumptions of polarization-sensitive imaging are explored, and refocusing of birefringent structures is experimentally demonstrated. PS-ISAM enables high-resolution volumetric imaging of birefringent materials and tissue. (C) 2015 AIP Publishing LLC.

[http://dx.doi.org/10.1063/1.4936236]
\end{abstract}

Modern advancements in optical physics continue to improve upon microscopy techniques, enabling imaging of thick samples. Optical coherence tomography (OCT) is a coherent optical imaging modality, which measures a broadband interferometric signal to reconstruct the threedimensional (3-D) structure of scattering samples. ${ }^{1}$ It provides cellular level resolution with imaging depths of $1-3 \mathrm{~mm}$ in scattering tissue. OCT has proven most useful in ophthalmology, where it is now part of the standard of care. ${ }^{2}$ In addition, it has been developed for application in cardiovascular, gastroesophageal, and cancer imaging. ${ }^{3}$ OCT has also found application beyond the medical field, in areas such as metrology, non-destructive testing, microfluidics, and others. ${ }^{4}$

As in other 3-D optical imaging modalities, OCT suffers from reduced depth-of-field when increasing the numerical aperture (NA) of the imaging system. Interferometric synthetic aperture microscopy (ISAM) is a solution to the coherent microscopy inverse problem and provides spatially invariant transverse resolution through an efficient Fourier domain coordinate transformation, similar to that in synthetic aperture radar. ${ }^{5}$ Because ISAM is a point-scanned technique, it does not suffer from the cross-talk common in full-field techniques. ${ }^{6}$ Additionally, it has been shown to be robust with respect to motion when using either high-speed scanning or motion correction techniques. ${ }^{7}$ ISAM can therefore be used for real-time, in vivo imaging with high transverse resolution throughout depth. ${ }^{7}$

Polarization-sensitive optical coherence tomography (PS-OCT) is a functional extension of OCT developed for probing the birefringence of materials and biological tissue. ${ }^{8}$ In addition to three-dimensional scattering structure, PSOCT measures the polarization state of the backscattered light. Imaging samples that exhibit birefringence will cause a change in the measured polarization state. In biological tissues, form birefringence arises due to the organization of the tissue microenvironment, which provides insight into various biological processes. In particular, PS-OCT has been particularly valuable for imaging disease in birefringent tissues such as muscle, skin, the retina, arterial plaque, and the breast. ${ }^{9}$ PS-OCT is also well-suited for the imaging of birefringent materials such as polymers. ${ }^{10}$

PS-OCT makes use of the Jones vector representation to model the imaging system. ${ }^{11}$ This formalism describes each polarization component of the optical system as a Jones matrix $\mathbf{J}$, which operates upon the electric field Jones vector $\mathbf{E}$. The form birefringence of the sample introduces a phase retardation between the two components of the Jones vector, which can be measured using PS-OCT. The use of the Jones calculus assumes the propagation of a collimated, or pencil, beam along the optic axis with the polarization restricted to the transverse plane. In practice, the sample is illuminated with a focused beam, which is taken into account by the ISAM model to provide high-resolution throughout depth. We propose the use of a hybrid model in the low-NA regime, abandoning the pencil beam approximation while retaining the transverse polarization approximation.

The power ratio between the transverse and longitudinal polarization components of the focused Gaussian beam is shown in Fig. 1. The focused vector beams were simulated for circularly polarized incident light for increasing NA. ${ }^{12}$ Initially, the power is confined to the transverse polarization. As the NA increases, more power is transferred to the longitudinal polarization. However, only at a very high numerical aperture is the longitudinal polarization strong enough to allow calculation of the longitudinal susceptibility component of the sample. ${ }^{13}$ In the low-NA regime where OCT imaging is typically performed ( $0.1 \mathrm{NA}$ or lower $\left.\mathrm{r}^{5,7}\right)$, the longitudinal polarization is greatly overwhelmed by the power 


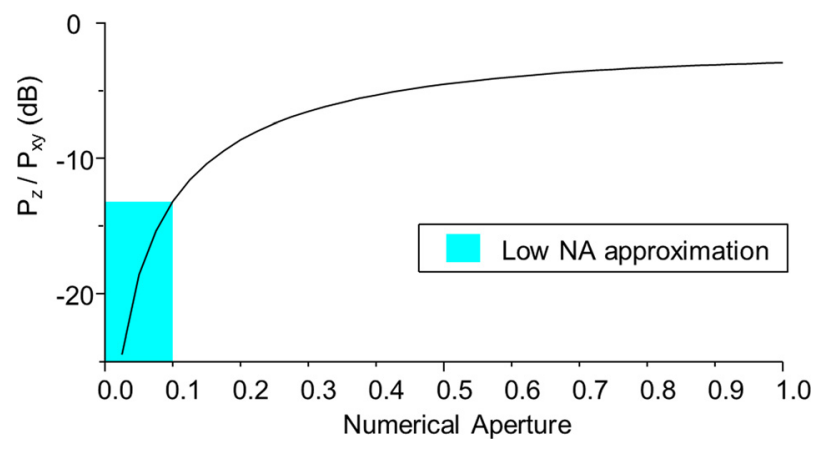

FIG. 1. Simulation of the longitudinal (z) and transverse (xy) beam power vs increasing numerical aperture for light circularly polarized prior to focusing.

in the transverse polarization. This supports the use of the Jones vector representation at low-NA as in the standard PSOCT model.

In general, ISAM imaging systems are identical to those used in OCT, with the exception that a higher NA may be used without sacrificing depth-of-field. Experimental data for this paper were acquired with a custom built spectral domain PS-OCT system using a traditional free-space PS-OCT design. ${ }^{8,9}$ The optical source was a super luminescent diode centered at $1300 \mathrm{~nm}$ with $100 \mathrm{~nm} 3 \mathrm{~dB}$ bandwidth (Thorlabs). Polarization-maintaining (PM) fiber was used to deliver light to and from the free-space PS-OCT interferometer through an in-line linear polarizer (Thorlabs ILP13010PM-APC), optical circulator (AFW Technologies PMP-13-R-C3N-45-22), and $45 \mathrm{~m}$ of PM fiber, which was included to displace the ghost images out of the imaging range. ${ }^{14}$ The collected interference signal returned through the circulator to a $\mathrm{PM}$ fiber polarization beam splitter (AC Photonics PBS-13-P-2-2-1-1) for polarization diverse detection. The two polarization components of the Jones vector were measured with spectrometers using 2048 pixel line scan cameras (Sensors Unlimited GL2048L). Both the axial and transverse resolution of the system were approximately $7.65 \mu \mathrm{m}$ full-width-half-maximum (FWHM), or $13 \mu \mathrm{m}\left(\frac{1}{e^{2}}\right)$, giving an NA of 0.065 , slightly greater than the $0.05 \mathrm{NA}$ of the initial ISAM demonstrations. ${ }^{5}$ Three-dimensional datasets were acquired by scanning $512 \times 256$ transverse points with an isotropic transverse sampling of $3.4 \mu \mathrm{m}$.

The ISAM reconstruction was applied to each component of the measured Jones vector through a Fourier domain coordinate resampling of the data according to the relationship

$$
k=\frac{1}{2}\left(Q_{x}^{2}+Q_{y}^{2}+Q_{z}^{2}\right)^{1 / 2}
$$

for wavenumbers $k=2 \pi n / \lambda$ and spatial frequencies $Q$, where $n$ is refractive index and $\lambda$ is wavelength. Following this step, the sample reflectivity $R$ and phase retardation $\delta$ were calculated for each position $(x, y, z)$ in the 3 -D volume as

$$
\begin{gathered}
R(x, y, z) \propto|H(x, y, z)|^{2}+|V(x, y, z)|^{2}, \\
\delta(x, y, z)=\arctan \left(\frac{|H(x, y, z)|}{|V(x, y, z)|}\right),
\end{gathered}
$$

where $H$ and $V$ are the horizontal and vertical components of the measured Jones vector, respectively. A two-dimensional
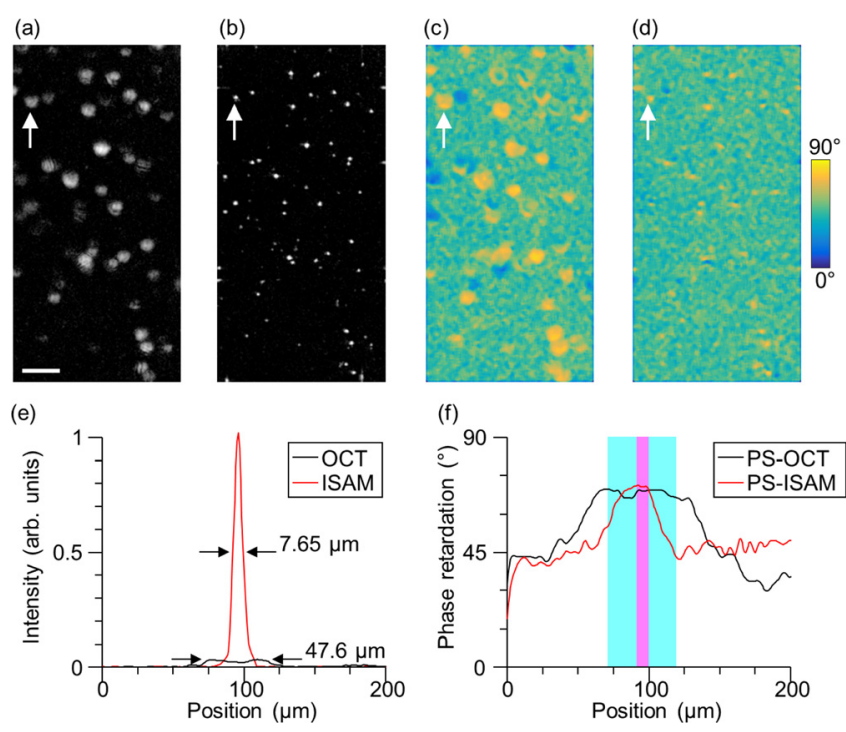

FIG. 2. Imaging of a silicone phantom consisting of sub-resolution microparticles. En face planes are taken from $1502 \mu \mathrm{m}$ optical distance above focus (10.5 Rayleigh ranges). (a) OCT intensity image. (b) ISAM intensity image. (c) PS-OCT phase retardation image. (d) PS-ISAM phase retardation image. (e) Trace of the OCT and ISAM intensities for a single particle (white arrow) showing the FWHM resolution. (f) Trace of the PS-OCT and PS-ISAM phase retardation for the same particle as in (e). Shaded cyan and magenta areas indicate regions of valid signal from OCT and ISAM intensity measurements respectively, determined from the FWHM measurements in (e). Scale bar indicates $200 \mu \mathrm{m}$.

median filter of approximately two resolution elements was applied to the phase retardation data to remove random fluctuations for improved visualization. The standard OCT structural data were given by $R$, while a change in the phase retardation indicated a change in the polarization state.

To demonstrate the PS-ISAM reconstruction, a scattering phantom consisting of $\mathrm{TiO}_{2}$ particles $(<5 \mu \mathrm{m})$ suspended in a silicone gel was imaged. The results are shown in Fig. 2. An en face plane taken from far above focus shows strong blurring in the OCT image (Fig. 2(a)) caused by the limited depth-of-field. The corresponding ISAM reconstruction shown in Fig. 2(b) shows clear improvement in the transverse resolution. PS-OCT and PS-ISAM phase retardation images are shown in Figs. 2(c) and 2(d). The valid polarization information is localized to areas with sufficient signal corresponding to the scattering particles in the intensity images, which have phase retardation values near the extremes of the scale corresponding to right- and left-handed polarization states. ${ }^{15}$ Comparison of the PS-OCT and PSISAM images reveals improved localization of the phase retardation information in the PS-ISAM reconstruction due to the improved transverse resolution. The traces for a single particle shown in Figs. 2(e) and 2(f) highlight the improvement of the ISAM and PS-ISAM reconstruction over the standard techniques.

Figure 3 demonstrates PS-ISAM imaging in a birefringent material. The phantom consisted of small molded plastic pieces suspended in an agarose gel. In dense scattering samples such as this, the improvement from OCT to ISAM may not be dramatic due to the relatively uniform scattering structure (Figs. 3(a) and 3(d)). However, the birefringence of the sample causes change in the polarization state seen as variations in the phase retardation images (Figs. 3(c) and 3(d)). 
(a)

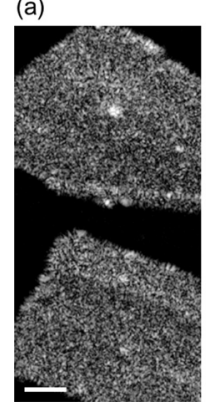

(b)

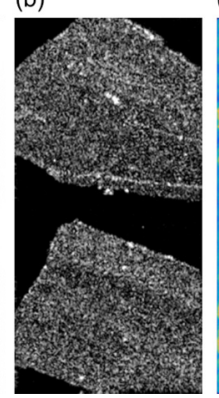

(c)

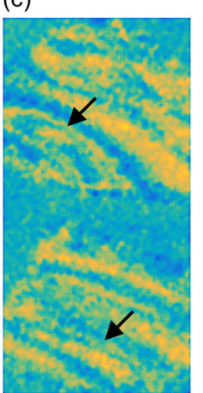

(d)

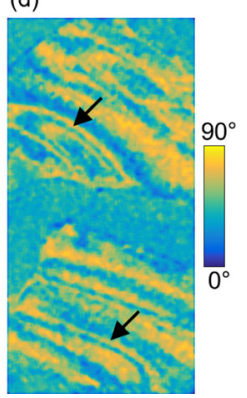

FIG. 3. Imaging of molded plastic pieces suspended in agarose gel. En face planes are taken from $1000 \mu \mathrm{m}$ optical distance above focus (7 Rayleigh ranges). (a) OCT intensity image. (b) ISAM intensity image. (c) PS-OCT phase retardation image. (d) PS-ISAM phase retardation image. Resolution of the phase retardation image is improved (highlighted by arrows). Scale bar indicates $200 \mu \mathrm{m}$.

This highlights the usefulness of polarization-sensitive imaging as an additional form of contrast. Due to the strong defocus, the phase retardation information in the PS-OCT image is somewhat indistinct. In contrast, the PS-ISAM reconstruction clearly resolves the polarization patterns throughout the material as a result of the improved transverse resolution.

To demonstrate the PS-ISAM technique in biological samples, healthy human breast tissue from a 24 year old female was imaged ex vivo. The focus was placed far below the tissue surface to increase signal collection from deep within the sample. ${ }^{7}$ The resulting cross-sectional images are shown in Fig. 4. The changes in the polarization state indicated by the phase retardation measurement (Figs. 4(c) and 4(d)) arise due to the aligned collagen fibers in the human breast. ${ }^{9}$ These features are more clearly resolved in the PSISAM reconstruction, showing fine structure not visible in the PS-OCT data. To quantitatively demonstrate the improvement in image quality, image metrics were used to assess the sharpness of the phase retardation images. The metrics were calculated for a region of interest in the breast tissue, marked in Fig. 4, which contained the changes in polarization state due to sample birefringence, while the entire field of view was included for assessing the birefringent plastic in Fig. 3. The PS-ISAM images for both the birefringent phantom and human tissue samples showed improvement over the PS-OCT images in the anisotropy 7,15 and Sobel ${ }^{16}$ image metrics of greater than $50 \%$ and $7.5 \%$, respectively. This improved image quality could potentially benefit the detection of residual cancer in breast tumor margins, where the polarization information can aid in the differentiation between normal and diseased tissue by identifying birefringent regions as collagenous stroma.

PS-ISAM is well-suited for optical imaging applications where both high transverse resolution and large imaging depth are desired. This work demonstrates the application of PS-ISAM in both birefringent materials and human tissue. In medicine and biology, the high resolution polarization information could be used to more accurately distinguish between disease and tissue types, providing insight into the spatial arrangement of the tissue microenvironment. The improved resolution of polarization patterns may also prove useful for visualization of stress distribution in materials testing.
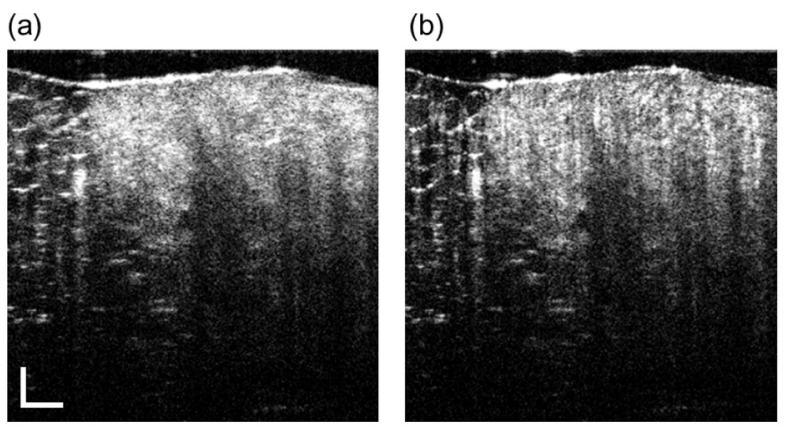

(c)

(d)
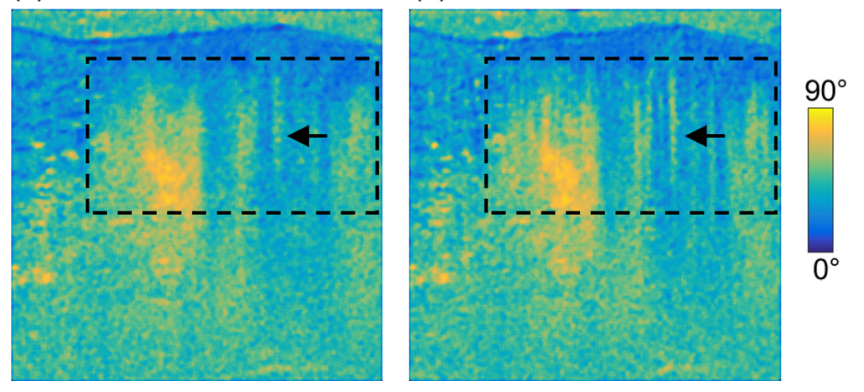

FIG. 4. Imaging of ex vivo human breast tissue. Cross-sectional planes with focus placed $1253 \mu \mathrm{m}$ optical distance below the surface (8.8 Rayleigh ranges). (a) OCT intensity image. (b) ISAM intensity image. (c) PS-OCT phase retardation image. (d) PS-ISAM phase retardation image. Image metrics calculated over the boxed region in (c) and (d). Resolution of the phase retardation image is improved (highlighted by arrows). Scale bar indicates $200 \mu \mathrm{m}$.

When imaging at low-NA, the proposed PS-ISAM reconstruction technique is capable of improved visualization of polarization information through a simple resampling step, which is well-suited for real-time processing. Both PS-OCT and ISAM processing have been previously implemented in real-time using graphics processing units. ${ }^{7,9}$ Although the data acquisition rate in PS-ISAM is twice that of traditional ISAM, it is likely that PS-ISAM may also be implemented for realtime processing and display through proper memory management. It is also possible that additional contrast could be obtained from the longitudinal component of the refractive index when performing optical tomography at increased NA. Future work will explore the theory and application of PSISAM in high-NA imaging of thick samples.

The authors thank Thorlabs $\mathrm{GmbH}$ for support in developing the PS-OCT system used in this study, and the National Disease Research Interchange for procuring the human tissue used in this study. This research was supported in part by Grants from the National Institutes of Health (1 R01 CA166309). P.S.C. acknowledges funding in part with Federal funds from the National Cancer Institute, National Institutes of Health, Department of Health and Human Services, under Contract No. HHSN261201400044C. S.A.B. and P.S.C. are co-founders of Diagnostic Photonics, Inc., which is licensing intellectual property from the University of Illinois at UrbanaChampaign related to interferometric synthetic aperture microscopy. S.A.B. has received patent royalties from the Massachusetts Institute of Technology for technology related to optical coherence tomography. Other authors declare no competing financial interests. Additional information can be found online at http://biophotonics.illinois.edu. 
${ }^{1}$ D. Huang, E. Swanson, C. Lin, J. Schuman, W. Stinson, W. Chang, M. Hee, T. Flotte, K. Gregory, C. Puliafito, and J. G. Fujimoto, Science 254, 1178 (1991); S. A. Boppart, B. E. Bouma, C. Pitris, J. F. Southern, M. E. Brezinski, and J. G. Fujimoto, Nat. Med. 4, 861 (1998).

${ }^{2}$ C. A. Puliafito, M. R. Hee, C. P. Lin, E. Reichel, J. S. Schuman, J. S. Duker, J. A. Izatt, E. A. Swanson, and J. G. Fujimoto, Ophthalmology 102, 217 (1995); W. Drexler, U. Morgner, R. K. Ghanta, F. X. Kärtner, J. S. Schuman, and J. G. Fujimoto, Nat. Med. 7, 502 (2001); W. Drexler and J. G. Fujimoto, Prog. Retinal Eye Res. 27, 45 (2008).

${ }^{3}$ J. A. Evans, J. M. Poneros, B. E. Bouma, J. Bressner, E. F. Halpern, M. Shishkov, G. Y. Lauwers, M. Mino-Kenudson, N. S. Nishioka, and G. J. Tearney, Clin. Gastroenterol. Hepatol. 4, 38 (2006); I.-K. Jang, G. J. Tearney, B. MacNeill, M. Takano, F. Moselewski, N. Iftima, M. Shishkov, S. Houser, H. T. Aretz, E. F. Halpern, and B. E. Bouma, Circulation 111, 1551 (2005); F. T. Nguyen, A. M. Zysk, E. J. Chaney, J. G. Kotynek, U. J. Oliphant, F. J. Bellafiore, K. M. Rowland, P. A. Johnson, and S. A. Boppart, Cancer Res. 69, 8790 (2009).

${ }^{4}$ D. Stifter, Appl. Phys. B 88, 337 (2007).

${ }^{5}$ T. S. Ralston, D. L. Marks, P. S. Carney, and S. A. Boppart, Nat. Phys. 3, 129 (2007); B. J. Davis, S. C. Schlachter, D. L. Marks, T. S. Ralston, S. A. Boppart, and P. S. Carney, J. Opt. Soc. Am. A 24, 2527 (2007); B. J. Davis, D. L. Marks, T. S. Ralston, P. S. Carney, and S. A. Boppart, Sensors 8, 3903 (2008).

${ }^{6}$ D. L. Marks, T. S. Ralston, S. A. Boppart, and P. S. Carney, J. Opt. Soc. Am. A 24, 1034 (2007); D. L. Marks, B. J. Davis, S. A. Boppart, and P. S. Carney, J. Opt. Soc. Am. A 26, 376 (2009); D. Hillmann, G. Franke, C. Lührs, P. Koch, and G. Hüttmann, Opt. Express 20, 21247 (2012).

${ }^{7}$ N. D. Shemonski, S. G. Adie, Y.-Z. Liu, F. A. South, P. S. Carney, and S. A. Boppart, Opt. Express 22, 19183 (2014); N. D. Shemonski, A. Ahmad, S. G. Adie, Y.-Z. Liu, F. A. South, P. S. Carney, and S. A. Boppart, Opt. Express 22, 19314 (2014); N. D. Shemonski, S. S. Ahn, Y.-Z. Liu, F. A.
South, P. S. Carney, and S. A. Boppart, Biomed. Opt. Express 5, 4131 (2014); N. D. Shemonski, F. A. South, Y.-Z. Liu, S. G. Adie, P. Scott Carney, and S. A. Boppart, Nature Photon. 9, 440 (2015); A. Ahmad, N. D. Shemonski, S. G. Adie, H.-S. Kim, W.-M. W. Hwu, P. S. Carney, and S. A. Boppart, Nat. Photonics 7, 444 (2013); Y.-Z. Liu, N. D. Shemonski, S. G. Adie, A. Ahmad, A. J. Bower, P. S. Carney, and S. A. Boppart, Biomed. Opt. Express 5, 2988 (2014).

${ }^{8}$ M. R. Hee, D. Huang, E. A. Swanson, and J. G. Fujimoto, J. Opt. Soc. Am. B 9, 903 (1992); J. F. de Boer, T. E. Milner, M. J. van Gemert, and J. S. Nelson, Opt. Lett. 22, 934 (1997).

${ }^{9}$ J. J. Pasquesi, S. C. Schlachter, M. D. Boppart, E. Chaney, S. J. Kaufman, and S. A. Boppart, Opt. Express 14, 1547 (2006); J. Strasswimmer, M. C. Pierce, B. H. Park, V. Neel, and J. F. de Boer, J. Biomed. Opt. 9, 292 (2004); E. Götzinger, M. Pircher, and C. K. Hitzenberger, Opt. Express 13, 10217 (2005); S. K. Nadkarni, M. C. Pierce, B. H. Park, J. F. de Boer, P. Whittaker, B. E. Bouma, J. E. Bressner, E. Halpern, S. L. Houser, and G. J. Tearney, J. Am. Coll. Cardiol. 49, 1474 (2007); F. A. South, E. J. Chaney, M. Marjanovic, S. G. Adie, and S. A. Boppart, Biomed. Opt. Express 5, 3417 (2014); R. Patel, A. Khan, R. Quinlan, and A. N. Yaroslavsky, Cancer Res. 74, 4685 (2014).

${ }^{10}$ D. Stifter, E. Leiss-Holzinger, Z. Major, B. Baumann, M. Pircher, E. Götzinger, C. K. Hitzenberger, and B. Heise, Opt. Express 18, 25712 (2010).

${ }^{11}$ R. C. Jones, J. Opt. Soc. Am. 31, 488 (1941); J. F. de Boer and T. E. Milner, J. Biomed. Opt. 7, 359 (2002).

${ }^{12}$ B. Richards and E. Wolf, Proc. R. Soc. A 253, 358 (1959).

${ }^{13}$ B. J. Davis and P. S. Carney, J. Opt. Soc. Am. A 25, 2102 (2008).

${ }^{14}$ M. K. Al-qaisi and T. Akkin, Opt. Express 18, 3392 (2010).

${ }^{15}$ A. Doronin, C. Macdonald, and I. Meglinski, J. Biomed. Opt. 19, 025005 (2014); J. A. Kubby, Adaptive Optics for Biological Imaging (CRC Press, 2013), pp. 200-202.

${ }^{16}$ S. Gabarda and G. Cristóbal, J. Opt. Soc. Am. A 24, B42 (2007). 